\title{
An Appraisal of Dual Antiplatelet Therapy with Clopidogrel and Aspirin for Prevention of Cardiovascular Events
}

\author{
Chris Terpening, PhD, PharmD
}

Combination antiplatelet therapy, typically with clopidogrel and aspirin, is commonly used for the prevention of cardiovascular events. When used for appropriate indications and duration, its benefits clearly outweigh its risks. However, it is not uncommon for the combination to be used outside of recommended indications or for longer than recommended durations. In these circumstances data are at best unclear and, at worst, indicative of harm. Furthermore, use for one of its indications-prevention of cardiac events after deployment of a coronary stent-is complicated by the type of stent used. This report reviews the evidence surrounding combination antiplatelet therapy with clopidogrel and aspirin, with an emphasis on identifying appropriate indications for and durations of therapy. ( $\mathrm{J}$ Am Board Fam Med 2009;22:51-56.)

Primary care physicians (PCPs) often find themselves in the situation in which a patient's cardiologist institutes therapy with clopidogrel in combination with low-dose aspirin, but then defers routine follow-up back to the PCP. This combination offers certain theoretical benefits. Platelet activation is a critical step in the formation of thrombotic clots. Aspirin inhibits the production of thromboxanes, which play a prominent role in platelet activation. Clopidogrel, a thienopyridine, acts by inhibiting adenosine receptors, which play a major role at a different step in platelet activation. Thus, their mechanisms are complementary and may decrease clot formation over either agent alone. Furthermore, resistance to the effects of each agent has been well reported, ${ }^{1}$ but resistance to both agents in a given patient should be less frequent. Although this combination of antiplatelet agents has been demonstrated to offer clinical benefits under certain circumstances, it does raise problems as well. Most significantly, the reiterative

This article was externally peer reviewed.

Submitted 20 December 2007; revised 3 July 2008; accepted 9 July 2008.

From the Departments of Family Medicine and Clinical Pharmacy, West Virginia University, Charleston Division.

Funding: none.

Conflict of interest: none declared.

Corresponding author: Chris Terpening, $\mathrm{PhD}$, PharmD, Departments of Family Medicine and Clinical Pharmacy, West Virginia University, WVU Health Sciences Center Charleston Division, 3110 MacCorkle Avenue SE, Charleston, WV 25304 (E-mail: cterpening@hsc.wvu.edu). platelet inhibition increases the likelihood of bleeding. Thus, it is incumbent on the PCP to understand the evidence behind the use of combination therapy and the point at which potential benefits may be offset by its risks. Only then can the PCP make an informed decision about when to return the patient to monotherapy with antiplatelets. Unfortunately, although the literature on this combination of agents is extensive, data regarding the benefits and risks of long-term use are often conflicting. Newer thienopyridines, such as prasugrel, seem to offer both greater benefit and greater risk and may confuse the situation further. ${ }^{2}$ This article will outline what is known and make reasonable recommendations for the PCP.

\section{Indications for Combination Therapy}

The primary determinant of using combination therapy is, of course, the indication. An overview of clinical trials investigating the efficacy of the combination of clopidogrel and aspirin has been provided (Table 1). These trials have identified some conditions where combination therapy offers no benefits over monotherapy. Combination therapy has been shown to be no more effective than aspirin alone in primary prevention of coronary or cerebral events in patients at high risk. ${ }^{3}$ Aspirin, at a dose of 75 to $162 \mathrm{mg}$ daily, is the preferred treatment for primary prevention; clopidogrel alone is useful in patients with an aspirin allergy. ${ }^{4}$ Likewise, combination therapy is inappropriate in patients with a 
Table 1. Efficacy of Clopidogrel Plus Aspirin in Selected Clinical Trials

\begin{tabular}{|c|c|c|c|c|c|}
\hline Trial & Patient Population & Endpoint & $\begin{array}{c}\text { Event Rate } \\
\text { Combination (\%) }\end{array}$ & $\begin{array}{c}\text { Event Rate } \\
\text { Monotherapy (\%) }\end{array}$ & $P$ \\
\hline CHARISMA (3) & CAD & $\begin{array}{l}\text { CV death, MI, or CVA at } 28 \\
\text { months }\end{array}$ & 6.8 & 7.3 & .22 \\
\hline MATCH (5) & CVA & $\begin{array}{l}\text { CVA, MI, CV death, or CV } \\
\text { hospitalization at } 18 \text { months }\end{array}$ & 15.7 & $16.7^{*}$ & .244 \\
\hline CLARITY (6) & STEMI & $\begin{array}{l}\text { Occlusion, death, or recurrent } \\
\text { MI at } 8 \text { days }\end{array}$ & 15 & 21.7 & $<.001$ \\
\hline CURE (9) & NSTEMI & $\begin{array}{l}\text { CV death, MI, or CVA at } 12 \\
\text { months }\end{array}$ & 9.3 & 11.4 & $<.001$ \\
\hline PCI-CURE (12) & $\begin{array}{l}\text { NSTEMI with } \\
\text { PCI }\end{array}$ & $\mathrm{CV}$ death or $\mathrm{MI}$ at 8 months & 8.8 & 12.6 & .002 \\
\hline CREDO (11) & PCI & $\begin{array}{l}\text { Death, MI, or CVA at } 12 \\
\text { months }\end{array}$ & 8.5 & 11.5 & .02 \\
\hline
\end{tabular}

${ }^{*}$ Clopidogrel monotherapy.

CHARISMA, Clopidogrel for High Atherothrombotic Risk and Ischemic Stabilization, Management, and Avoidance; MATCH, Management of Atherothrombosis with Clopidogrel in High-Risk Patients; CLARITY, Clopidogrel as Adjunctive Reperfusion Therapy; CURE, Clopidogrel in Unstable Angina to Prevent Recurrent Events; PCI-CURE, Percutaneous Coronary Intervention in the Clopidogrel in Unstable Angina to Prevent Recurrent Events; CREDO, Clopidogrel for the Reduction of Events During Observation; CAD, coronary artery disease; CVA, cardiovascular accident; STEMI, ST-segment elevation myocardial infarction; NSTEMI, non-ST-segment elevation myocardial infarction; PCI, percutaneous coronary intervention; CV, cardiovascular; MI, myocardial infarction.

recent stroke or transient ischemic attack because it increases the incidence of major and minor bleeds without offering any therapeutic benefit over clopidogrel alone. ${ }^{5}$ The most appropriate indications for the use of combined clopidogrel and aspirin therapy are the treatment of acute coronary syndromes and the prevention of coronary events after placement of a stent.

\section{Acute Coronary Syndromes}

For patients who suffer an ST-segment elevation myocardial infarction and who do not receive stent placement in the course of treatment, current evidence only supports short-term use of combination therapy (roughly 1 to 2 weeks). ${ }^{6,7}$ Longer duration of use has not been addressed in these patients, although recent guideline updates consider combination clopidogrel and aspirin therapy reasonable for up to a year. ${ }^{8}$ Patients with unstable angina or non-ST-segment elevation myocardial infarction have stronger evidence of benefit from long-term therapy. ${ }^{9}$ Treatment with combination therapy for an average duration of 9 months lowered the incidence of cardiovascular death, myocardial infarction, and stroke at 12 months to $9.3 \%$ as compared with $11.4 \%$ in patients receiving aspirin alone. It should be noted that a significant proportion of patients subsequently received some form of revascularization (balloon angioplasty, stent placement, or coronary artery bypass graft) during their initial hospitalization, but results remained similar regardless of whether the procedure was performed or not.

\section{Coronary Stents}

Combination therapy is particularly important in the patient receiving coronary stents. It is used for a minimum of 28 days peri- and post-procedurally to lower the incidence of acute $(<24$ hour) and subacute ( 1 to 30 days) stent thrombosis. ${ }^{10}$ Furthermore, patients receiving clopidogrel plus aspirin for 1 year after a percutaneous coronary intervention (PCI) had a combined incidence of death, myocardial infarction, or stroke of $8.5 \%$, compared with $11.5 \%$ in patients receiving combination therapy for 28 days followed by aspirin alone. ${ }^{11}$ This corroborated and extended a substudy of the aforementioned unstable angina trial. ${ }^{12}$ Patients in that trial undergoing PCI received combination therapy for 1 month and then resumed their randomized therapy for an average of 8 months. This resulted in a combined incidence of cardiovascular death or myocardial infarction of $8.8 \%$ at the end of follow-up in those receiving combination therapy compared with $12.6 \%$ in those receiving aspirin alone.

These trials were conducted before the advent of drug-eluting stents. Sirolimus-eluting stents were 
introduced in the United States in 2003, with paclitaxel-eluting stents following in 2004. Their use was then very quickly and extensively adopted. This has complicated the debate about the appropriate duration of combination antiplatelet therapy considerably because the same mechanism that underlies the drug-eluting stents' benefits also imparts long-term complications.

Originally, stents were developed to reduce the incidence of acute and subacute reocclusion at the site of balloon angioplasty. This is different from the acute and subacute thrombosis that dual antiplatelet therapy helps to prevent. Thrombosis is mediated by disruption of the plaque and exposing the necrotic core, the contents of which stimulate platelet activation. Reocclusion occurs because of both elastic recoil of the artery and vascular thickening and contraction caused by scar formation. The permanent solid structure of the stent minimizes these complications. However, bare metal stents allow the intima to regrow through the structure. If this neointimal growth is excessive it can result in restenosis at the site. Restenosis does not generally result in acute events, but can cause the return of anginal symptoms and impact quality of life to the point where a repeat procedure must be performed.

Drug-eluting stents address this problem by exuding chemicals that inhibit neointimal growth, thus significantly reducing restenosis and subsequent revascularization procedures. However, that neointimal growth helps provide stability to the underlying plaque. Without it, the window for thrombosis caused by exposed plaque extends beyond the 1-month period typical for bare metal stents to at least 3 to 6 months. Failure to comply with dual antiplatelet therapy for at least this period of time is one of the largest risk factors for in-stent thrombosis in those receiving drug-eluting stents. Estimates of the hazard ratio from premature discontinuation of dual antiplatelet therapy range from $13.74^{13}$ to $89.78 .{ }^{14}$ Furthermore, postmortem pathology studies have shown re-endothelialization of the vessel may be incomplete even years after the insertion of a drug-eluting stent. ${ }^{15}$ This may or may not reflect a direct effect of the eluted drug. The drugs are lipophilic and thus may be retained for some time at the site. However, it also may reflect an inflammatory response to the nonadsorbable polymer. Either way, it does present a theoretical rationale for indefinite use of dual antiplate- let therapy in patients receiving drug-eluting stents.

Recently, a large number of publications have addressed the incidence of very late $(>1$ year after procedure) stent thrombosis with drug-eluting stents. They range from single registry data ${ }^{16}$ to large meta-analyses of clinical trials. ${ }^{17}$ Although the results are not entirely concordant, there seems to be a small excess of very late events when compared with bare metal stents. Interestingly, given the medically acute and serious nature of thromboses, there is not a clear trend toward increased myocardial infarction or death, although a few studies have reported this. In response to this data, the Food and Drug Administration ${ }^{4}$ and the American College of Cardiology/American Heart Association ${ }^{18}$ both issued guidance suggesting the use of dual antiplatelet therapy for "at least 1 year" in patients receiving drug-eluting stents. They stopped short of recommending indefinite therapy, in large part because it is not known if longer-term therapy will reduce the incidence of very late thromboses or myocardial infarctions.

\section{Benefits of Long-Term Use of Combination Therapy}

What scant literature there is on the use of clopidogrel and aspirin for longer than 1 year has yielded inconsistent findings. Results supporting long-term use of combination therapy for patients receiving drug-eluting stents were reported in a study from Duke Medical Center. ${ }^{19}$ In this observational trial, patients who took combination therapy for at least 12 months, most of whom continued therapy thereafter, had no deaths or nonfatal myocardial infarctions in the subsequent 12 months. Another group who stopped clopidogrel before 12 months but continued aspirin had a $4.5 \%$ adjusted rate of this composite endpoint between 12 and 24 months after their stent placement. There was no difference in second-year event rates in patients who received bare metal stents $(4.7 \%$ with an initial 12 months of clopidogrel vs $3.6 \%$ without). In opposition to these findings, 2 largescale registries of drug-eluting stent patients suggested that late use or discontinuation of clopidogrel did not effect late stent thrombosis. Twenty-three percent ${ }^{20}$ to $50 \%{ }^{13}$ of late thromboses occurred in the presence of combined antiplatelet therapy. Thus, although there is a theoretical 
Table 2. Bleeding Rates with Long-Term Combination Therapy in Selected Clinical Trials

\begin{tabular}{llcrr}
\hline & & & \multicolumn{2}{c}{ Major and Minor Bleeds } \\
\cline { 3 - 5 } Trial & Patient Population & Duration (months) & Combination Therapy (\%) & Monotherapy (\%) \\
\hline CURE (9) & NSTEMI & 9 & 8.8 & 5.1 \\
CREDO (11) & PCI & 12 & 14.1 & 12.3 \\
CHARISMA (3) & CAD & 28 & 3.8 & 2.6 \\
MATCH (5) & CVA & 18 & 5.1 & $1.6^{*}$ \\
TRITON (2) & ACS with PCI & 15 & $5.0^{\dagger} / 3.8$ & NA \\
\hline
\end{tabular}

${ }^{*}$ Clopidogrel monotherapy.

${ }^{\dagger}$ Prasugrel plus aspirin.

CURE, Clopidogrel in Unstable Angina to Prevent Recurrent Events; CREDO, Clopidogrel for the Reduction of Events During Observation; CHARISMA, Clopidogrel for High Atherothrombotic Risk and Ischemic Stabilization, Management, and Avoidance; MATCH, Management of Atherothrombosis with Clopidogrel in High-Risk Patients; TRITON, Trial to Assess Improvement in Therapeutic Outcomes by Optimizing Platelet Inhibition with Prasugrel; NSTEMI, non-ST-segment elevation myocardial infarction; CAD, coronary artery disease; CVA, cardiovascular accident; ACS, acute coronary syndrome; PCI, percutaneous coronary intervention.

rationale, there are no clear trends in the published evidence to support long-term dual antiplatelet therapy in the drug-eluting stent patient.

Data regarding long-term use in a broader population of patients are also somewhat conflicting. As noted earlier, the Clopidogrel for High Atherothrombotic Risk and Ischemic Stabilization, Management, and Avoidance trial ${ }^{3}$ showed no benefit from long-term use (28 months) of combination therapy in a high-risk cardiovascular patient population. However, an interesting contrast was reported in a secondary analysis of the data. ${ }^{21} \mathrm{~Pa}$ tients with stable cardiovascular disease but without a documented thrombotic event derived no benefit from reducing the combined endpoint of cardiovascular death, myocardial infarction, or stroke over receiving aspirin alone, with even a suggestion of harm (event rate, $6.3 \%$ vs $5.7 \%$ ). Conversely, if patients had a previous myocardial infarction, combination therapy did seem to reduce the subsequent incidence of events relative to aspirin monotherapy (6.6\% vs $8.3 \%)$. Both groups had significant numbers of patients with a prior PCI, although neither time from PCI nor proportion of stent type was reported.

\section{Bleeding Risks with Combination Therapy}

Although there is no consensus as to the benefit of long-term dual antiplatelet therapy, there is a general conclusion that it does pose bleeding risk. For instance, a case-control study evaluating patients with serious gastrointestinal bleeding suggested adjusted hazard ratios of 1.1 for clopidogrel mono- therapy, 1.8 for aspirin monotherapy, and 7.4 for their combination when compared with no treatment. ${ }^{22} \mathrm{~A}$ second similarly designed study reported adjusted hazard ratios of $1.67,1.39$, and 3.90 , for clopidogrel, aspirin, and their combination, respectively. ${ }^{23}$ In terms of absolute numbers, a study including 4 major combination therapy trials yielded an aggregate rate of severe bleeds of $1.8 \% .^{24} \mathrm{~A}$ review of those trials also yields additional minor bleeding with a range of $2.1 \%$ to $5.3 \%$. The total aggregate event rates for selected trials has been provided in Table 2. As mentioned earlier, a new thienopyridine, prasugrel, could prove even more problematic. ${ }^{2}$ In the TRITON study, prasugrel plus aspirin for 15 months yielded a $2.4 \%$ rate of major bleeds and $2.6 \%$ rate of additional minor bleeds. In the same study, clopidogrel plus aspirin caused a $1.8 \%$ rate of major bleeds and a $2.0 \%$ rate of minor bleeds. Unfortunately, the time course of the bleeding events in any of these trials was not clearly reported. Possibly, a subset of sensitive patients was selected out fairly early on with the remainder having little increased risk. ${ }^{21}$ However, this remains conjecture in the absence of well-designed trials.

\section{Conclusions}

Based on these data, there are some logical recommendations that can be offered (Table 3). Patients treated for acute coronary syndromes without the use of a stent should receive combination clopidogrel and aspirin for at least 1 month, and it is reasonable to lengthen that up to 1 year. Patients 
Table 3. Recommendations for the Use of Clopidogrel with Aspirin

\begin{tabular}{llc}
\hline Indication & Duration & SORT \\
\hline $\begin{array}{l}\text { Primary prevention of } \\
\quad \text { cardiovascular events }\end{array}$ & Avoid & B \\
Secondary prevention of stroke & Avoid & B \\
ACS, without stent & $1-12$ months & A \\
Bare-metal stent & $1-12$ months & A \\
Drug-eluting stent & 12 months & C \\
\hline
\end{tabular}

SORT, strength of recommendation taxonomy, ${ }^{25}$ ACS, acute coronary syndrome.

who receive stents, either electively or emergently, are not as clear-cut. Given the recent controversies, the use of bare metal stents may increase. This is probably appropriate but means that general assumptions as to the type of stent placed cannot be made. Although the primary care physician is not usually involved in choosing the type of stent to be placed, it is vital that the PCP find out this information. For patients receiving bare metal stents, combination therapy should be strongly recommended for the first month. Continued therapy out to 1 year may be helpful, but durations longer than that are not supported. For patients receiving drugeluting stents, 1 year of combination therapy should be encouraged. However, longer term therapy from that point may best be reserved for those with a clear prothrombotic history (ie, previous myocardial infarction) and a relatively low risk of bleeding.

Unfortunately, there is no standard definition as to who is at low risk of bleeding. There are certain patients who are clearly at high risk. These include patients with a personal history of significant bleed, advanced age, thrombocytopenia, recent stroke or transient ischemic attack, or those taking chronic anticoagulants or nonsteroidal anti-inflammatory drugs. Other factors that may contribute to bleeding include low body weight, alcoholism, poorly controlled hypertension, and impaired renal function.

Indefinite use of the combination of clopidogrel and aspirin should not be the rule at this time. The data in patients receiving drug-eluting stents sometimes show benefit but are far from compelling. For patients not receiving these stents, data are sparse and suggest little, if any, benefit. Long-term trials are needed to ascertain if any reduction in cardiovascular events is of sufficient magnitude to offset increases in the risk of bleeding. Until such time, PCPs should feel comfortable discontinuing clopidogrel after 1 year in many, if not most, instances.

\section{References}

1. Gladding P, Webster M, Ormiston J, Olsen S, White H. Antiplatelet drug nonresponsiveness. Am Heart J 2008;155:591-9.

2. Wiviott SD, Braunwald E, McCabe CH, et al. Prasugrel versus clopidogrel in patients with acute coronary syndromes. N Engl J Med 2007;357:2001-15.

3. Bhatt DL, Fox KAA, Hacke W, et al. Clopidogrel and aspirin versus aspirin alone for the prevention of atherosclerotic events. N Engl J Med 2006;354: 1706-17.

4. Fraker TD Jr, Fihn SD, Gibbons RJ. 2007 chronic angina focused update of the ACC/AHA 2002 Guidelines for the management of patients with chronic stable angina: a report of the American College of Cardiology/American Heart Association Task Force on Practice Guidelines Writing Group to develop the focused update of the 2002 Guidelines for the management of patients with chronic stable angina. Circulation 2007;116:2762-72.

5. Diener H-C, Bogousllovsky J, Brass LM, et al. Aspirin and clopidogrel compared with clopidogrel alone after recent ischaemic stroke or transient ischaemic attack in high-risk patients (MATCH): randomised, double-blind, placebo-controlled trial. Lancet 2004;364:331-7.

6. Sabatine MS, Cannon CP, Gibson CM, et al. Addition of clopidogrel to aspirin and fibrinolytic therapy for myocardial infarction with ST-segment elevation. N Engl J Med 2005;352:1179-89.

7. Chen ZM, Jiang LX, Chen YP, et al. Addition of clopidogrel to aspirin in 45,852 patients with acute myocardial infarction: randomised placebo-controlled trial. Lancet 2005;366:1607-21.

8. Antman EM, Hand M, Armstrong PW, et al. 2007 Focused Update of the ACC/AHA 2004 Guidelines for the Management of Patients with ST-Elevation Myocardial Infarction: a report of the American College of Cardiology/American Heart Association Task Force on Practice Guidelines: developed in collaboration with the Canadian Cardiovascular Society endorsed by the American Academy of Family Physicians: 2007 Writing Group to Review New Evidence and Update the ACC/AHA 2004 Guidelines for the Management of Patients with ST-Elevation Myocardial Infarction, writing on behalf of the 2004 Writing Committee. Circulation 2008;117: 296-329.

9. Yusuf S, Zhao F, Mehta SR, et al. Effects of clopidogrel in addition to aspirin in patients with acute coronary syndromes without ST-segment elevation. N Engl J Med 2001;345:494-502.

10. Bertrand ME, Rupprecht H-J, Urban P, Gershlick 
AH, CLASSICS Investigators. Double-blind study of the safety of clopidogrel with and without a loading dose in combination with aspirin compared with ticlopidine in combination with aspirin after coronary stenting: the Clopidogrel Aspirin Stent International Cooperative Study (CLASSICS). Circulation 2000;102:624-9.

11. Steinhubl SR, Berger PB, Mann JT 3rd, et al. Early and sustained dual oral antiplatelet therapy following percutaneous coronary intervention. A randomized controlled trial. JAMA 2002;288:2411-20.

12. Mehta SR, Yusuf S, Peters RJ, et al. Effects of pretreatment with clopidogrel and aspirin followed by long-term therapy in patients undergoing percutaneous coronary intervention: the PCI-CURE study. Lancet 2001;358:527-33.

13. Airoldi F, Colombo A, Morici N, et al. Incidence and predictors of drug-eluting stent thrombosis during and after discontinuation of thienopyridine treatment. Circulation 2007;116:745-54.

14. Iakovou I, Schmidt T, Bonizzoni E, et al. Incidence, predictors, and outcome of thrombosis after successful implantation of drug-eluting stents. JAMA 2005; 293):2126-30.

15. Finn AV, Nakazawa G, Joner $M$, et al. Vascular responses to drug eluting stents: importance of delayed healing. Arterioscler Thromb Vasc Biol 2007; $27: 1500-10$.

16. Lagerqvist B, James SK, Stenestrand U, et al. Longterm outcomes with drug-eluting stents versus baremetal stents in Sweden. N Engl J Med 2007;356: 1009-19.

17. Kastrati A, Mehilli J, Pache J, et al. Analysis of 14 trial comparing sirolimus-eluting stents with baremetal stents. N Engl J Med 2007;356:1030-9.

18. Grines CL, Bonow RO, Casey DE Jr, et al. Prevention of premature discontinuation of dual antiplate- let therapy in patients with coronary artery stents: a science advisory from the American Heart Association, American College of Cardiology, Society for Cardiovascular Angiography and Interventions, American College of Surgeons, and American Dental Association, with representation from the American College of Physicians. Circulation 2007;115: $813-8$.

19. Eisenstein EL, Anstrom KJ, Kong DF, et al. Clopidogrel use and long-term clinical outcomes after drugeluting stent implantation. JAMA 2007;297:159-68.

20. Daemen J, Wenaweser P, Tsuchida K, et al. Early and late coronary stent thrombosis of sirolimus-eluting and paclitaxel-eluting stents in routine clinical practice: data from a large two-institutional cohort study. Lancet 2007;369:667-78.

21. Bhatt DL, Flather MD, Hacke W, et al. Patients with prior myocardial infarction, stroke, or symptomatic peripheral areterial disease in the CHARISMA trial. J Am Coll Cardiol 2007;49:1982-8.

22. Hallas J, Dall M, Andries A, et al. Use of single and combined antithrombotic therapy and risk of serious upper gastrointestinal bleeding: population based case-control study. BMJ 2006;333:726-30.

23. Delaney JA, Opatrny L, Brophy JM, Suissa S. Drugdrug interactions between antithrombotic medications and the risk of gastrointestinal bleeding. CMAJ 2007;177:347-51.

24. Verheugt FW, Clappers N. Incidence of severe bleeding with long-term clopidogrel-aspirin combination: comparison with chronic warfarin therapy [abstract]. Circulation 2007;116(suppl II):441.

25. Ebell MH, Siwek J, Weiss BD, et al. Strength of recommendation taxonomy (SORT): a patient-centered approach to grading evidence in the medical literature. J Am Board Fam Pract 2004;17:59-67. 